\title{
Fisiopatología de la G. N. A. y su implicancia en el tratamiento
}

\author{
F. PLGA *, N. ALLENDE *, y L. REBOLLEDO (O. F.) **.
}

Los signos y síntomas de la GNA derivan de una alteración de la mernbrana filtrante del gloméruTo provocada por una "inflamación" aséptica del riñón, con depósito de complemento, IgG y antígenos del Estreptococo (1-2). Las etapas de esta inflamación han sido analizadas al microscopio de luz, desde los inicios de la enfermedad (3-4), apareciendo el glomérulo con un aspecto compacto, con disminución o desaparición de los lúmenes capilares y marcada reducción del espacio de Bowman, debido a la tumefacción y exudación del ovillo con escaso compromiso del túbulo e intersticio renales.

Esta inflamación es de corta duración y de remisión espontánea alrededor de las 4 semanas (4) y puede persistir una discreta proliferación glomerular, la que no tiene repercusión funcional medible.

Como signos clínicos más destacados se presentan el edema en un $97 \%$, la hipertensión en un $93 \%$ y la hematuria macroscópica en el $75 \%$ de los pacientes (5).

Los mecanismos fisiopatológicos posibles de producción de los dos prineros signos son el motivo de esta presentación con lo cual se puede implicar el tratamiento de los primeros días de estos enfermos que hace algunos años tenían una mortalidad entre el 2 y $5 \%$ y que actualmente es mucho menor.

Material y método. a 5 niños con GNA postestreptocócica se les practicó medición al ingreso, quinto $\mathrm{y} / \mathrm{o}$ décimo días del volumen plasmático con azul de Evans, flujo plasmático renal mediante el \% de extracción del Hipuran I 131, Clearence de Creatina endógena, excreción urinaria de $\mathrm{Na}, \mathrm{K}$ y diuresis en 24 horas. Además se hizo medición de la ingestión de $\mathrm{Na}$ y control de Ja Natremia.

Todos los pacientes tuvieron hjpertensión arterial y algunos con evidentes signos de insuficiencia cardíaca.

\footnotetext{
* Depto, Nefrourología, Hosp. L, Calvo Mackenna.
}

* Oulmico-Farmacéutico, Hosp. L. Calvo Mackenna.
Los dos primeros días se indicó una dieta hipoproteica y sin sal, con restricción de líquidos. Desde el $5^{\circ}$ día régimen normal sin sal hasta los 10 días cuando se agregó sal normalmente. Todos permanecieron en reposo en cama.

Los cinco pacientes curaron definitivamente cerca de los 6 meses.

Resultados. Flujo sanguíneo renal: Figura No 1. Como en todo proceso inflamatorio, en los primeros días de la nefritis, el flujo está aumentando para alcanzar niveles normales a los 10 días (6-7). La técnica usada consistió en determinar el \% de extracción del Hipuran I 131 a los 5 minutos, encontrándose un $72 \%$ de extracción en el primer día y de $52 \%$ a los 10 y 20 días.

Clearence de creatina endógena: Figura Nọ 2. E1 compromiso de la filtración glomerular es acentuado en el primer día, alcanzando un promedio de $56,9 \mathrm{ml} / \mathrm{mi} / 1.73 \mathrm{~m}^{2}$, pero se recupera cerca

Fiujo sunguineo renal: fepuración de Hlpurán $I_{1 x}$ en el Jp, 10. 20 dias de G. N. A. - (7 ninos).

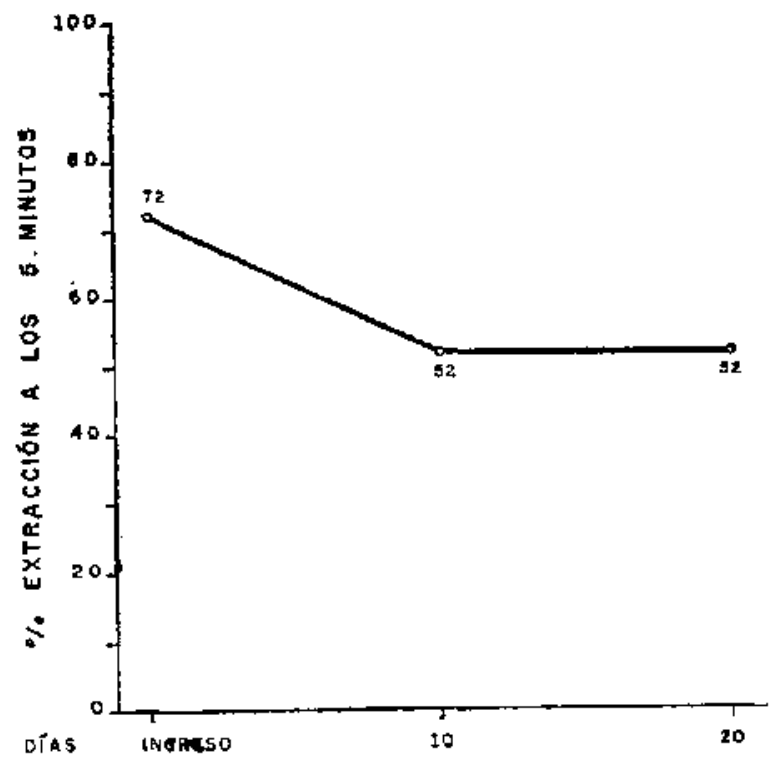

Fig. No 1 
de los 10 días de enfermedad (promedio 102,6 $\mathrm{ml} / \mathrm{min} / 1,73 \mathrm{~m}^{2}$ ). Diuresis y osmolaridad: Figura No 3. Hay una reducción de la diuresis en los primeros dias (prom. $344 \mathrm{ml} / 24$ hrs.), pero la osmolaridad es alta, lo que indica que probablemente la capacidad tubular es normal en este sentido. Al $5^{\circ}$ día do la diuresis aumenta y la osmolaridad urinaria baja, dada la poliuria y a la dieta hipoproteica y sin sal. Excreción de $N a$ y $K$

Depuración promedio de Creatinina Endogena, en el to $y$ 10: dins de G. N. A. - (5 nifos).

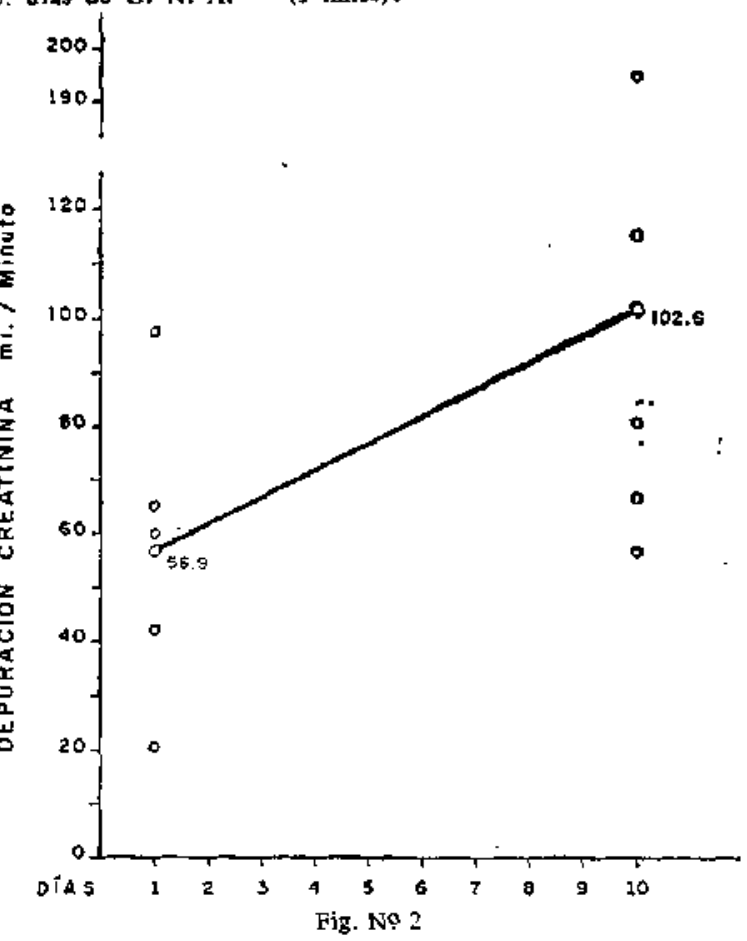

Promedlo de Dluresis y Osmolarkdad en orina de 24 horas, en los d(as 1?, 5: y 10 ? de G. N. A. - (5 nlfios).

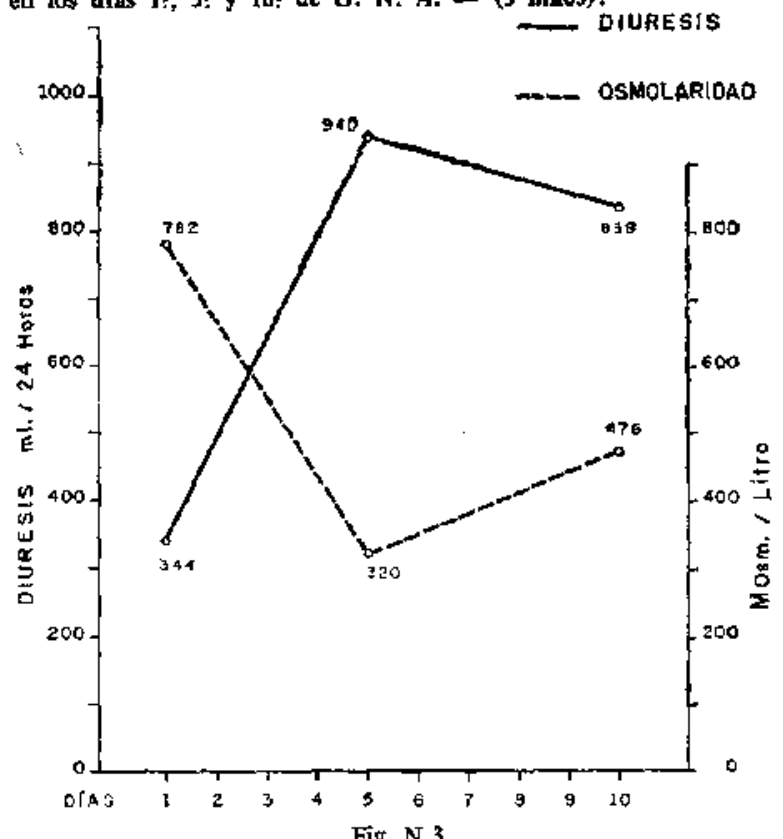

en 24 hrs.: Figura No 4. El primer dia el Na está reducido máxime si consideramos que todo edema revela aumento del contenido total de sal y agua. Al 5ọ día hay una importante eliminación de $\mathrm{Na}$ $(79,2 \mathrm{mEq} / 24 \mathrm{hrs}$ ) en relación con cl aumento de la diuresis por fusión del edema, con baja de peso del paciente. El $\mathrm{K}$ se mantiene entre 20,4 a $36,04 \mathrm{mEq} / 24 \mathrm{~h}$. en la orina hasta los 10 días.

Natremia: Figura No 5 . Esta se mantiene invariable a pesar de las grandes variaciones en la ingestión de $\mathrm{Na}$, agua y diuresis en el 1, $10 \mathrm{y}$ 20 días lo que revela que el control tubular de este catión sería normal. Volumen piasmático total: Figura No 6. Se aprecia una plétora que alcanza a un aumento de más del $100 \%$ del volumen plasmático total medido con la técnica de dilución de Azul de Evans (8-9). A los diez dias, este volumen se ha reducido sin alcanzar aún los niveles normales para la superficie corporal de estos niños.

Presión venosa: Elevada al ingreso se normaliza al $10^{\circ}$ día.

Presión arterial: Elevada en todos los niños al ingreso, se normaliza simultáneamente con la normalización de Ia P. V. y desaparición del edema clínico.

Comentario. La etapa inicial de la GNA tenía un riesgo mortal del 1 al $5 \%$ en estadísticas de hace algunos años. La mujor comprensión de la fisiopatología ha permitido reducir este riesgo a cero de mortalidad.

El punto ac partida de la enfermedad es una inflamación glomerular aséptica por un proceso

Excrecion udonria de Ne $\mathrm{g} K$ en 24 horas, en los dias 19 , 5: 10? de G. N. A. - (5 nlnos).

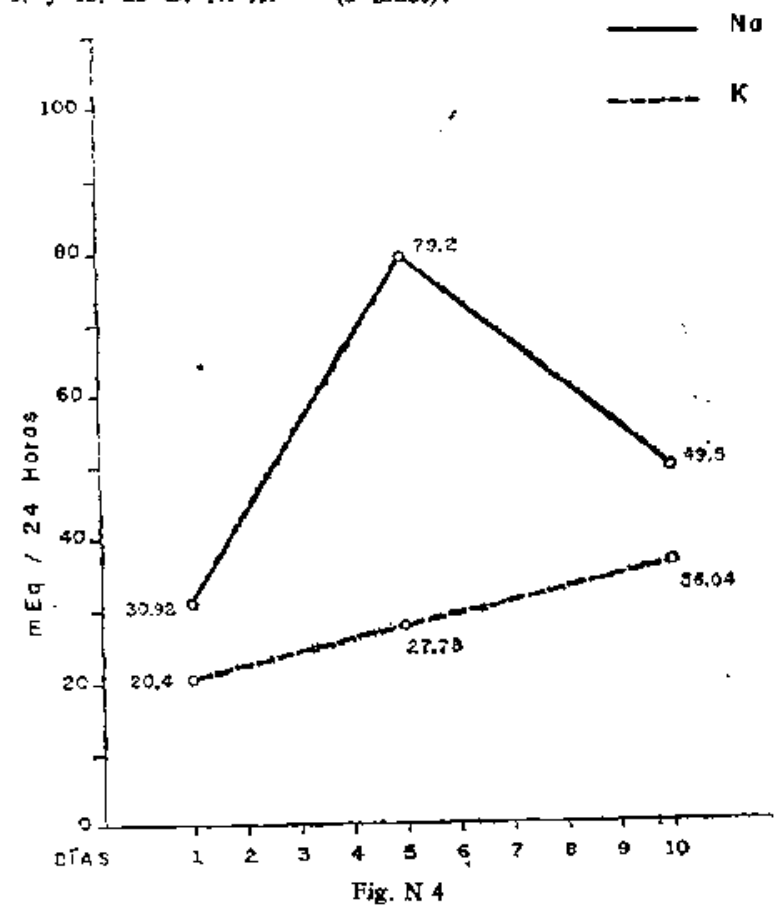




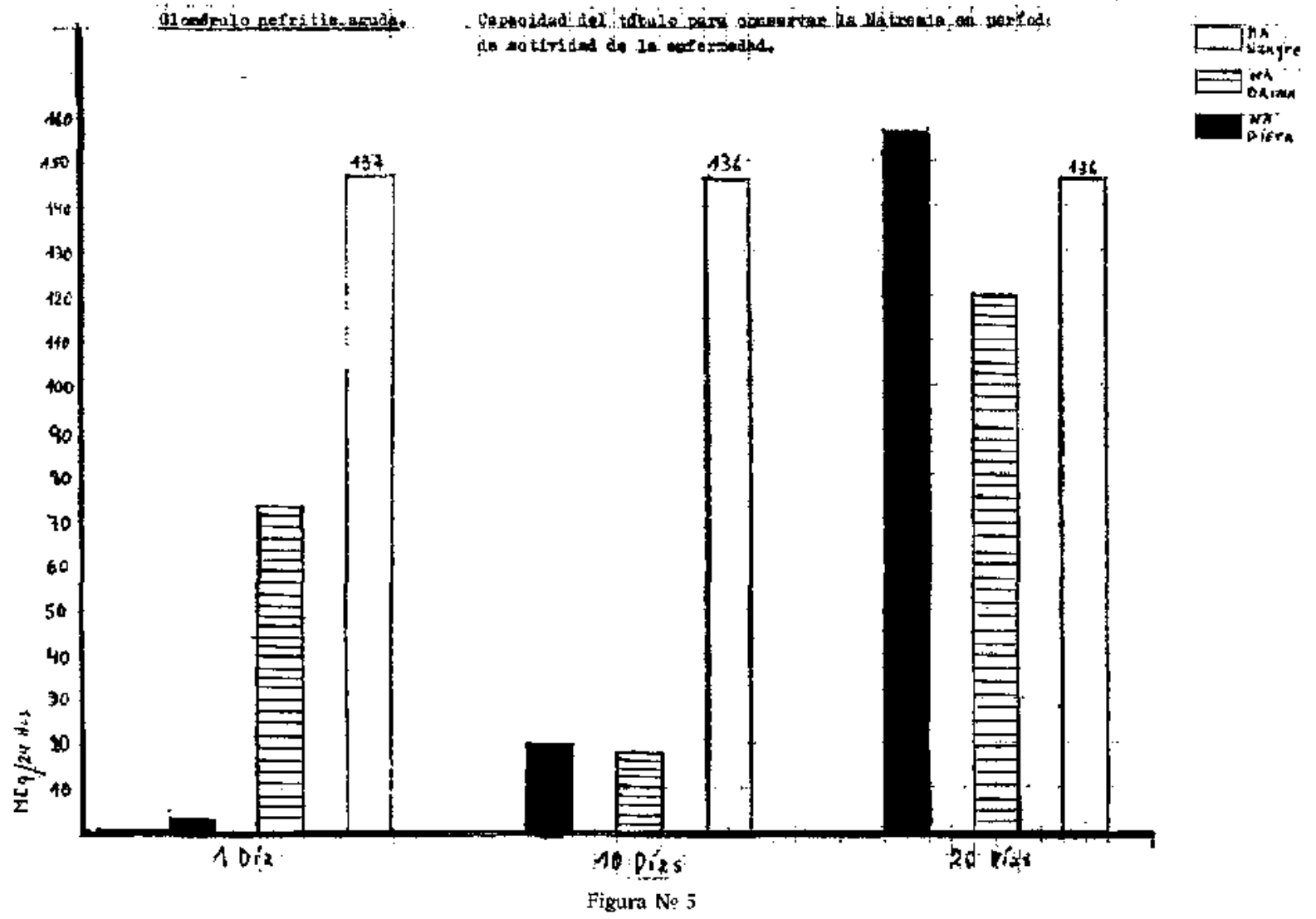

Promediog de volumen sanguineo, plasmitico (Azul de Erans) p presión yenosa, al te y 10 ? días de G. N. A. - (5 nthos).

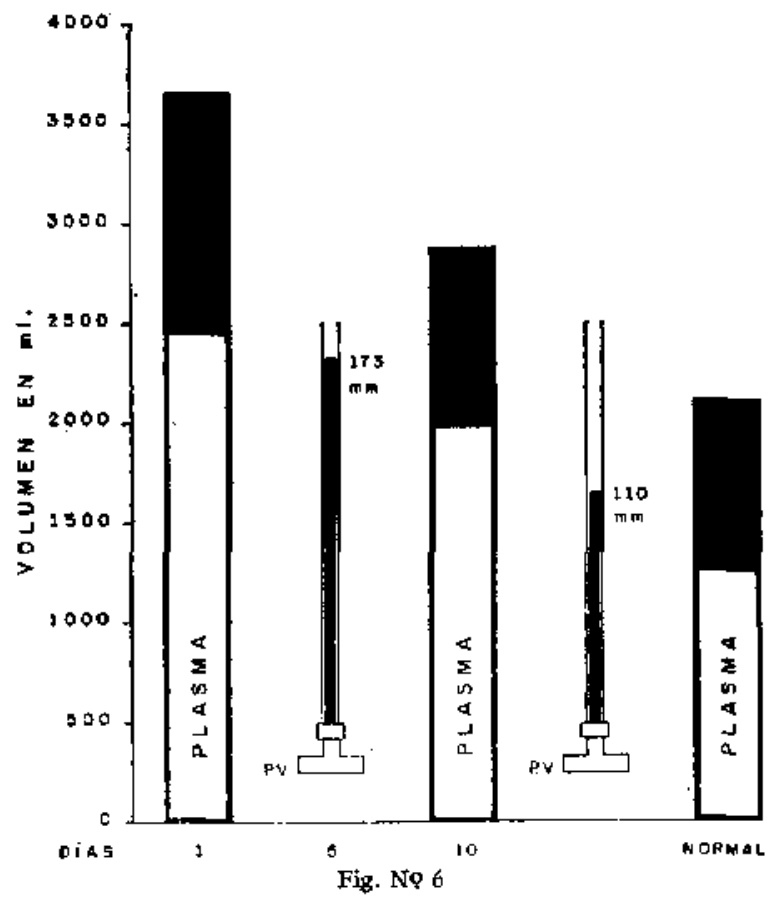

autoinmunitario de las membranas filtrantes. Esto trae como consecuencia una disminución de la eliminacion renal de agua y sales (oliguria) favo- reciendo la aparición de un balance positivo que conduce a una plétora del espacio extracelular y en especial del intravascular.

La caída de la filtración glomerular es de un $56 \%$ con consecuencia aumento de la volemia en más de un $100 \%$ del considerado normal para la superficie corporal de estos pacientes.

Esta hipervolemia intravascular sería un factor joportante en la génesis de la hipertensión arterial, condicionante de las complicaciones graves en el comienzo de la nefritis (P. A. Debito) (1011). El otro factor es el mecanismo de renina-angiotensina, regulador del diámetro arteriolar el que a su vez estaría regulado por las variantes del flujo del $\mathrm{Na}$ en la mácula densa del túbulo distal. La relación de causa efecto de la plétora e hipertensión podría ser exacta por el hecho de que ésta desaparece simultáneamente con aquella.

EI aumento de volumen intravascular agudo conduce a un aumento de la presión venosa central, aumentando la perfusión cardíaca. El corazón responde a este aumento de flujo venoso con dilatación de sus cavidades (12), mejor rendimiento de su contractilidad y crecimiento del débito minuto, taquicardia. Sin embargo esta capacidad de adaptación no es ilimitada. Nuevo aumento de la perfusión se traducirá en una acentuada reducción del débito minuto (Starling) con la consiguiente plétora hacia atrâs (edema pulmonar y/o hepatomegalia $\mathrm{y}$ otros signos congestivos). 
Esta insuficiencia cardíaca no es consecuencia de daño cardíaco (12), sino que exclusivamente es dada por el aumento del volumen vascular de aparición aguda.

Otro efecto de la plétora intravascular es el aumento de la presión venosa central. Este auaumento del volumen ha de ser severo ya que es conocida la capacidad de adaptación del continente venoso a las variaciones del contenido, sin que se aprecie variaciones importantes de la presión venosa (13). Para el equilibrio del volumen del espacio extracelular se requiere que la cantidad de líquidos que salen desde el intravascular al intersticial sean iguales al que retorna al intravascular (14). Este intercambio se efectúa principalmente al nivel capilar y por diferencias de presión entre ellos. En el lado capilar arterial sale líquido y nutrientes por la gradiente favorable al intravascular que determina la presión arterial sobre la intersticial. Esta gradiente llega a cero a medida que avanza la corriente por el capilar y luego se invierte la presión favorable para el retorno hacia el intravascular. Esto ocurre en condiciones normales. Pero, cuando aumenta la presión venosa, presión que también se trasmite hacia los capilares, esta gradiente de retorno se reduce impidiendo el regreso de líquidos al espacio vascular, con to que aumenta en forma exagerada la circulación linfática, la que no es capaz de transportarla apareciendo el edema. Así, nuevamente, la plétora vascular, a través del aumento de la presión venosa central es la causa del edema.

Podemos concluir que en la génesis de la hipertensión arterial, el edema, la insuficiencia cardíaca y la hipertensión endocraneana hay un factor común que es la plétora circulatoria producida por un balance positivo de agua y sales debido a una caída de la filtración glomerular dañada por una inflamación de la membrana filtrante.

De esta conclusión podemos colegir que el tratamiento de la GNA debe estar dirigido fisiopatológicamente a reducir este balance positivo. Con este fin se han usado distintos métodos:

a) control en la ingestión de líquidos y sales (15).

b) Secuestro o extracción activa de líquidos circulantes mediante torniquetes, sangrías y dialisis con soluciones hipertónicas.

c) Diuréticos, en especial la Furosemide cuyo efecto es tan extraordinatio que ha desplazado a los métodos anteriores (16).

\section{RESUMEN}

Se analizan posibles mecanismos fisiopatológicos para el edema, la hipertensión arterial y las complicaciones clinicas de la Glomerulo Nefritis estreptocbicica en 5 pacientes.
Se puede concluir que ol daño inflanatorio de la membrana filtrante glomerular conduce a un balance de agua y sales, produciéndose una plétora del volumen plasmático, plétora que irtervendría en la génesis de la presión arterial y del edema. Se analizan esquemas terapéuticos hasados en estos restitados.

\section{SUMMARY}

The physiopathologic causes of hipertension, edema and clinical complication of Glomerulo Nephritis are analized in five patients from the first through the tenth diny.

Renal blood flow is raised at the begining with the G. F. R. reduced causing oliguria with a high osmolarity during the first day producing a positive balance of water and salt, racing the plasma volume to over a $100 \%$ the normal, simultaneusly with a high central venous pressure, considered to be one of the causes of edema.

The plasma volume is reduced at the tenth day but still over normal levels, coincident with normal blood pressure and disappearence of edema.

It is concluded that treatment in the first day of A. G. N. should be mainly directed to produce a negative balance of salt and water, obtained by restricted diet and Furosemid lised in hig dosis.

\section{BIBLIOGRAFIA}

1.-- Mcliors R. C. y Orsega L. C. Anialytical Patholo. gy. New observations on the pathogencsis of Glomerulo Nephritis, etc. Amer. J. Path 32: 55, 1956.

2.-- Acute Glomerulo Nephritis. Editor Jack Metcoff, Pag. 69. J. y A. Churchill I.td. Londres 1967.

3.- Id. Pág. 165.

4.- Paga F. La biopsia renal en la glomírulo-nefritis aguda. Rəv. Chil. Ped, 2: 81, 1966.

5.- Nefrología. Editor J. Howard. Editorial Andrés Bello. 1968. Prig. 74.

6.- Parrish A. E. Rubenstein N. H. y Howe J. S. Correlation between renal function and hystology Amer. J. Med. Sci. 229: 632, 1955.

7.-Reabi. F. Traite du Neftologie.

8.-Repetto G. Pediatría. 11: 44, 1968. Stgo. Chile.

9.-Cardozo E. L. Hydremia in acute nephritis. Acta Med. Scand. 125: 333, [946.

10.-Pirga F. Allende N. Maguire H. Estudio Hipertensión en niño. Rev, Chil. Ped. 42: 37, 1971.

11.-Guyton A. C. Textbook of medical Physiology. Pág. 209. Editor W. B. Saunders Co. 1971.

12.-Id. Pág. 345.

13.- Id. Pág. 214.

14.-Id. Pág. 233.

15.-Nefrología. Editor J. Howard. Pág. 82. Edit. Andrés Bello.

16.- Paredes S. Puga F. Allende N. Furosemide en el tratamiento de la I Cardíaca de la GNA. Rev. Chil. Ped. 41: 1043, 1970. 